\section{PSICOLOGÍA IBEROAMERICANA}

\section{Psicología Iberoamericana}

ISSN: 1405-0943

psicología.iberoamericana@uia.mx

Universidad Iberoamericana, Ciudad de México

México

Vizzuetth Herrera, Aleyda; García Meraz, Melissa; Guzmán Saldaña, Rebeca María Elena

Construcción y validación de dos escalas para usuarios de redes sociales virtuales:

conductas sexuales de riesgo y motivación hacia el sexo en línea

Psicología Iberoamericana, vol. 23, núm. 1, enero-junio, 2015, pp. 66-74

Universidad Iberoamericana, Ciudad de México

Distrito Federal, México

Disponible en: http://www.redalyc.org/articulo.oa?id=133944230008

Cómo citar el artículo

- Número completo

- Más información del artículo

- Página de la revista en redalyc.org

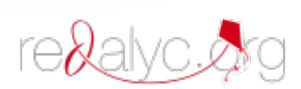

Sistema de Información Científica

Red de Revistas Científicas de América Latina, el Caribe, España y Portugal Proyecto académico sin fines de lucro, desarrollado bajo la iniciativa de acceso abierto 


\title{
Construcción y validación de dos escalas para usuarios de redes sociales virtuales: conductas sexuales de riesgo y motivación hacia el sexo en línea
}

\author{
Construction and validation of two scales for users virtual social networks: \\ Risky sexual behaviors and motivation for online sex
}

Aleyda Vizzuetth Herrera

Melissa García Meraz

Rebeca María Elena Guzmán Saldaña

Universidad Autónoma del Estado de Hidalgo

\section{RESUMEN}

En la actualidad no se cuenta con un instrumento validado capaz de medir las conductas sexuales de riesgo y la motivación hacia el sexo en línea en español y para población mexicana, de tal modo que se desarrollaron dos escalas a través de un estudio de campo de tipo transversal. Las escalas se administraron a 263 participantes (edad: 16 a 26 años, media: 23.20; hombres: 146; mujeres: 117). Se realizaron análisis factoriales y de fiabilidad. A partir de las conductas sexuales de riesgo se encontraron dos dimensiones ( $87 \%$ de la varianza explicada y alfa 0.962 ). Finalmente, la escala de motivación quedó conformada por una dimensión (68.93\% de la varianza explicada y alfa 0.909). Los resultados se discuten en función de la cultura, la sexualidad de hombres y mujeres y las normas asociadas al género.

Palabras Clave: conductas sexuales de riego, redes sociales virtuales, sexo, desarrollo de escalas, cibercultura

\section{ABSTRACT}

Currently no validated instrument exists that is capable of measuring risky sexual behavior and motivation for online sex in Spanish for the Mexican population. Therefore, two scales were applied using a transversal field study. The scales were applied to 263 participants (aged between 16 and 26, average: 23.20; men: 146; women: 117). Factor and reliability analyses were carried out. Two aspects were revealed for risky sexual behaviors $(87 \%$ of the explained variation and alpha 0.962$)$. Finally, the motivation scale consisted of a dimension (68.93\% of the explained variance, and alpha 0.909 ). The results are discussed on the basis of culture, the sexuality of men and women, and gender-related norms associated.

Keywords: Risky sexual behaviors, virtual social networks, sex, development of scales, cyber culture. 
De acuerdo con Gordon (2009), la mitad de los adolescentes que utilizan redes sociales virtuales, tales como MySpace, han posteado información acerca de su conducta sexual, abuso de sustancias e, incluso, violencia interpersonal. Estos datos ubican al grupo de adolescentes en alto riesgo de sufrir algún evento de violencia sexual; así como acoso, cyberbullying o ciberacoso y otras situaciones (Kujath, 2011).

Cuando hacemos referencia a conductas sexuales de riesgo es común que imaginemos aspectos relacionados con las relaciones sexuales sin protección. Sin embargo, en los últimos años, se destaca el uso de las redes sociales con el objetivo de recibir mensajes de tipo sexual, publicar fotografías de desnudos (propios y de otros) así como contactar extraños (Baumgartner, Valkenburg \& Peter, 2010; Crimmins \& SiegfriedSpellar, 2014; Lau \& Yuen, 2013; Wright \& Randall, 2012). Al considerar las conductas sexuales de riesgo en línea o en ambientes virtuales se abre todo un campo de investigación poco abordado en México y que coloca a la población usuaria de redes sociales virtuales en una alta situación de vulnerabilidad. Dado la poca investigación realizada se planteó la necesidad de contar con instrumentos válidos y confiables capaz de medir dichas conductas a la población mexicana. El artículo comienza con una descripción de instrumentos validados en otros países, seguido de la construcción y validación de un instrumento para los habitantes de este país.

\section{Instrumentos de conductas sexuales de riesgo en internet}

Se considera que la principal preocupación sobre los riesgos sexuales en internet se reduce a dos ideas: 1) ser víctima de una insinuación sexual indeseada $y$ 2) engancharse activamente en conductas sexuales en línea (Baumgartner, Valkenburg \& Peter, 2010). Las insinuaciones sexuales indeseadas, conductas sexuales con riesgo y su diferencia entre jóvenes y adultos, fueron los temas en que los investigadores holandeses centraron para su estudio. Ellos trabajaron con 1765 jóvenes (entre los 12 y 17 años) y 1026 adultos (entre 18 y 88 años) holandeses. Los adolescentes fueron divididos en tres grupos para representar el desarrollo de la adolescencia temprana (12-13 con una $\mathrm{N}=568$, $\mathrm{M}=12.49$ años, $\mathrm{SD}=0.51)$, la adolescencia media
(14-15 años con una $\mathrm{N}=606, \mathrm{M}=14.49$ años, $\mathrm{SD}=$ 0.50 ), y la adolescencia tardía ( $16-17$ con una $\mathrm{N}=591$, $\mathrm{M}=16.46$ años, $\mathrm{SD}=0.50$ ). Los adultos también fueron divididos en tres grupos el primero de 18 a 29, y refleja la adultez emergente $(\mathrm{N}=171, \mathrm{M}=24.00$ años, $\mathrm{SD}=3.21)$; el segundo grupo refleja la adultez media (30 a 50 años, $\mathrm{N}=416, \mathrm{M}=40.15$ años, $\mathrm{SD}=6.08$ ) y el tercero, la adultez tardía (mayores de 50: $\mathrm{N}=439$, $\mathrm{M}=63.99$ años, $\mathrm{SD}=8.85$ ). La insinuación sexual no deseada fue abordada mediante dos preguntas. Las categorías de respuesta iban de 0 (nunca), hasta 4 (seis veces o más). Los dos reactivos construidos mostraron una fuerte correlación y fueron: ¿qué tan frecuentemente, en los pasados seis meses, alguien con quien hablabas en línea te preguntó acerca de sexo cuando tú no lo deseabas? y ¿qué tan frecuentemente, en los pasados seis meses, alguien con quien hablabas en línea te solicitó hacer algo sexual que tú no deseabas?

De igual manera Baumgartner, Valkenburg y Peter (2010) preguntaron acerca de si en los pasados seis meses los participantes habían presentado alguna de las siguientes actividades: 1) buscar a alguien en línea para hablar de sexo, 2) buscar a alguien por internet para tener sexo, 3) enviar por internet una fotografía o video, en el cual ellos aparecieran parcialmente desnudos a alguien que conocieran únicamente en línea. Las categorías de respuesta iban de 0 (nunca), hasta 4 (seis veces o más). Finalmente, se agregaron cuatro reactivos que evalúan la percepción de riesgo en línea y un reactivo sobre la percepción de beneficio. Las categorías de respuesta tienen un rango de 0 (para nada peligroso) a 4 (muy peligroso). Las preguntan fueron: ¿qué tan peligroso es buscar en internet a una persona para hablar de sexo?, ¿qué tan peligroso es buscar en internet a alguien para tener sexo?, ¿qué tan peligroso es enviarle a alguien que conoces únicamente por internet fotos o videos en los cuales tú estés parcialmente desnudo?, ¿qué tan peligroso es enviarle a alguien tu dirección o teléfono cuando sólo lo conoces en línea?, y ¿qué tan benéfico consideras que es buscar por internet a alguien para tener sexo? Esta dimensión obtuvo un alfa de 0.83 .

\section{Texting y sexting}

Otra dimensión relacionada a las conductas sexuales de riesgo hace referencia al envío de mensajes con con- 
tenido de sexo explícito. En el 2012, Drouin y Landgraff notaron la importancia que cobra el envío de mensajes de texto entre los jóvenes, tanto como forma de fortalecer sus relaciones personales con amigos, como por ofrecer una oportunidad de aumentar su círculo social para conocer gente nueva. Sin embargo, también ha traído consigo la atención de las autoridades estadounidenses, ya que el aumento en las conductas sexuales con riesgo entre los jóvenes es alarmante en cuanto a la distribución de pornografía ilegal

Drouin y Landgraff (2012) realizaron un estudio sobre el texting, el sexting y el apego en las relaciones románticas de los estudiantes. Trabajaron con una muestra de 744 estudiantes universitarios (233 hombres, 511 mujeres). Sólo se trabajó con estudiantes que manifestaron haber mantenido una relación de pareja formal de entre 4 meses y 2 años. El instrumento está dividido en una parte demográfica, acerca del uso de las redes sociales (qué tipo de redes usan); la segunda parte está formada por un instrumento para determinar el grado de apego que mantuvieron en su relación de pareja, tomando 12 reactivos del instrumento Experiences in Close Relationships Scale el cual contiene reactivos tales como "trato de mantenerme realmente cerca de mi pareja”. El modo de respuesta es en una escala tipo Likert de 7 puntos (1-fuertemente en desacuerdo a 7-fuertemente de acuerdo) el Alfa que muestra el instrumento fue de 0.85 .

Para evaluar la dimensión de sexting y texting se les pidió a los participantes que contestaran qué tan frecuentemente enviaban mensajes de texto, videos y fotografías con contenido sexual a sus parejas. Se les solicitó que contestaran pensando en su pareja formal más representativa, es decir, un novio/a, el modo de respuesta fue en una escala tipo Likert de 6 puntos (0-nunca, a 5-muy frecuentemente).

Benotsch, Kalichman y Cage (2002) realizaron un estudio sobre el sexting, el uso de sustancias y las conductas sexuales con riesgo en jóvenes. Considerando el sexting como una conducta sexual de riesgo que aumenta su gravedad al vincularla con el uso de sustancias entre los jóvenes. Los autores trabajaron con un total de 800 participantes universitarios de entre 18 y 25 años, a los cuales se les pidió que contestaran una serie de preguntas demográficas. El instrumento se dividió en tres dimensiones que abarcaban el uso de sustancias donde se les preguntó sobre la frecuencia en el consumo de sustancias como marihuana, alcohol, éxtasis, cocaína entre otras, durante los últimos tres meses. Trabajaron con la dimensión de conducta sexual preguntando por el total de parejas sexuales en los últimos tres meses, así como el total de actos sexuales sin protección en ese periodo.

Para la dimensión de texting y sexting se les pidió a los participantes que contestaran el número estimado de mensajes de texto que reciben en un día normal en una escala de 10 puntos $(0 ; 1-15 ; 16-30 \ldots$ 106-120; más de 120). Además, se le pidió a los participantes que mencionaran si alguna vez habían practicado el sexting y, de ser así, el número total de imágenes que han enviado y recibido, así como el número de veces que han tenido sexo con alguien por primera vez después de que practicaron el sexting con esa persona.

Finalmente, Drouin, Vogel, Surbey y Stills (2013), siguiendo con el trabajo sobre sexting, realizaron una investigación para profundizar más sobre las prácticas de esta conducta con una muestra de 253 estudiantes (105 hombres y 148 mujeres) de entre 18 y 26 años. En el estudio se trabajó con siete dimensiones a evaluar, primero con una identificación de la relación que los participantes habían tenido, al solicitarles que contestaran pensando en su relación con una persona con quien estaban comprometidos, con una persona con quien habían mantenido una relación informal, y con una persona con quien le habían sido infiel a su pareja formal. Se trabajó en la dimensión de prevalencia del sexting, con un modo de respuesta en escala tipo Likert (1-nunca, a 6-muy frecuentemente). De igual manera, trabajaron con una dimensión llamada contenido de los mensajes de fotos y videos, pidiendo a los participantes que indicaran el contenido de los mensajes que enviaban, si eran fotografías de cuerpos desnudos o en posturas sugerentes. Finalmente, preguntaron por la razón por la que enviaban los mensajes con respuestas como: coqueteo, mi pareja me lo pidió, avergonzada de hacerlo cara a cara, ambos lo deseaban, aburrimiento, la pareja estaba lejos, otro.

Las mujeres, los adolescentes con problemas, los usuarios frecuentes de internet, los participantes en salas de chat, y todos aquellos que se comunican en línea con extraños presentan un mayor riesgo de recibir solicitudes de sexo en línea (Mitchell, Finkelhor \& 
Wolak, 2001). Además, en otras investigaciones se ha reportado que un $25 \%$ de los jóvenes han recibido por internet, material sexual explícito sin haberlo solicitado (Mitchell, Finkelhor \& Wolak, 2001).

Livingston (2003) establece que la mayor preocupación sobre las conductas de riesgo en internet debería centrarse en que los jóvenes son acosados por indeseado e inapropiado contacto sexual de adultos extraños. Tomando en cuenta que el tiempo de exposición semanal dedicado a internet es de 12.4 horas por cada internauta mexicano, esto significa que el periodo frente a una computadora es mayor al dedicado a la radio, la televisión, los periódicos impresos e incluso a convivir con los amigos. No sería de extrañarse que 8 millones de los usuarios de internet lo usen para buscar pareja en páginas tales como Match.com y otras redes sociales (La Jornada, 2011). Considérese que Facebook tiene 734 millones de usuarios y México es el país en tercer lugar de usuarios de redes sociales.

En la actualidad, no se cuenta con un instrumento en México encargado de investigar las conductas sexuales con riesgo que pueden presentar las personas a través de las redes sociales virtuales tales como Facebook, Twitter, Skype o WhatsApp (entre otras). Los instrumentos existentes encargados de evaluar este tipo de conductas son principalmente estadounidenses y europeos aunque en México no se cuenta con la traducción y validación de ninguno de ellos.

\section{MÉTODO}

Con base en las dimensiones trabajadas en los diferentes cuestionarios sobre conductas sexuales en línea, se consideraron de importancia para la elaboración del instrumento las siguientes dimensiones: "comportamiento sexual social en línea" de Delmonico y Miller (2003) "insinuaciones sexuales no deseadas", "conductas sexuales con riesgo", "percepción de riesgo", "percepción de beneficios", "comunicación por internet" de Baumgartner, Valkenburg y Peter (2010); "sexting y texting” de Drouin y Landgraff (2012) y Benotsch, Kalichman y Cage (2002) y, finalmente, "medio de uso para enviar mensajes sexuales", "riesgo asociado con el envío de mensajes sexuales", "contenido de los mensajes" "motivos de envío" de Drouin, Vogel, Surbey y Stills (2013), adicionalmente a las dimensiones de "cibersexo", "flirteo", "envío de imágenes con contenido sexual", "envío de videos con contenido sexual" obtenidas en los grupos focales tanto virtuales como presenciales elaborados previamente para el desarrollo de esta investigación. Es importante mencionar que actualmente se le considera a todo envío de imágenes, videos, o texto con contenido sexual como "sexting", sin embargo, para un mejor entendimiento del cuestionario, se trabajó con reactivos individuales para cada una de las conductas (imágenes, videos, mensajes).

Se realizó un estudio de campo de tipo transversal con una población compuesta por jóvenes, porque son la población que se encuentra en mayor peligro de presentar conductas sexuales con riesgo (Ybarra, Espelage \& Mitchell, 2007). Por esa razón, basamos nuestro estudio en esta población.

\section{a) Objetivo específico}

Construir y validar un instrumento que mida las conductas sexuales de riesgo que realizan los usuarios de redes sociales virtuales en interacciones que describen como formales así como los motivos por las cuales las ejecutan.

\section{b) Participantes}

Se trabajó con una muestra no probabilística de 263 usuarios de redes sociales. Las edades de los participantes se encuentran entre 16 y 50 años, con una media de 23.20 (117 mujeres, 55 estudiantes de preparatoria y 62 universitarias; así como 146 hombres, 68 estudiantes de preparatoria y 78 universitarios).

Los participantes fueron reclutados de las redes sociales así como de blogs y foros dedicados a la búsqueda de pareja. En total se obtuvo la participación voluntaria de 836 participantes, de los cuales sólo 236 declararon abiertamente utilizar las redes sociales con motivos de búsqueda de encuentro sexuales. Por ello, se decidió trabajar con esta muestra. Cabe mencionar que en México se considera que existen 35 millones de internautas (AMIPCI, 2014).

\section{c) Variables}

Definición conceptual de conducta sexual con riesgo en línea. Se consideran como conducta sexual con riesgo el inicio temprano de las relaciones sexuales, las múltiples parejas sexuales, la falta de uso o la inconsistencia en el uso de preservativos, abuso en la 
pornografía, acoso (Malamuth, Addison, \& Koss 2001; Bogaert, 2001; Fisher \& Barak, 1991; Benotsch, Kalichman \& Cage, 2002). En el caso de las conductas sexuales de riesgo en línea se consideran como aquellas relacionadas con ser víctima de una solicitud sexual en línea no deseada y ser involucrado en una conducta sexual en línea, como compartir fotos de desnudos o recibirlas (Mitchell, Finkelhor \& Wolak, 2001, 2007; Mitchell, Finkelhor \& Wolak, 2007a; Ybarra, Espelage \& Mitchell, 2007).

Por ello, las conductas sexuales de riesgo en línea pueden ser definidas como el engancharse de manera activamente en actividades sexuales en línea que pueden tener consecuencias negativas para el usuario, que van desde la decisión activa de participar en ellas, hasta recibir solicitudes de otros (Baumgartner, Vlakenburg \& Peter, 2010).

\section{d) Procedimiento}

Se construyó y validó una batería de instrumentos que miden las conductas sexuales con riesgo que ejecutan los usuarios de redes sociales virtuales en relaciones formales, así como los motivos asociados. Se aplicó de manera virtual y presencial el instrumento de Conductas Sexuales de Riesgo Dadas en Usuarios de Redes Sociales Virtuales a 263 participantes. Se les aseguró anonimato y confidencialidad para el manejo de los resultados. Se trabajó con estudiantes de preparatoria y licenciatura.

\section{e) Instrumento}

Se consideraron tres dimensiones para el instrumento: "conductas sexuales en línea", "sexting-imágenes", "sexting-videos". Así como una escala para evaluar motivos acerca de este tipo de conductas en relaciones formales. Se redactaron 14 reactivos según lo reportado por la literatura y un estudio previo con grupos focales. Se trata de una escala tipo Likert con cinco opciones de respuesta desde nunca hasta muy frecuentemente. Además, se redactaron preguntas sociodemográficas, así como frecuencia en el uso de las redes sociales virtuales.

La dimensión "conductas sexuales en línea" la constituyeron cuatro reactivos tales como: he recibido solicitudes para tener alguna conducta sexual por internet, he utilizado nombres sexualizados o sugerentes en chats en línea. La dimensión sexting-imágenes está for- mada por siete reactivos tales como: he enviado fotografías personales con contenido sexual, he enviado fotografías personales con desnudos. Finalmente, la dimensión sexting-videos cuenta con tres reactivos tales como: he enviado videos personales con contenido sexual, he mantenido cibersexo en vivo a través de videoconferencias (por ejemplo vía Skype).

La escala para evaluar motivos cuenta con cinco reactivos tales como: envié algún mensaje de texto o imagen o video con contenido sexual para coquetear: envié algún mensaje de texto o imagen o video con contenido sexual por aburrimiento a x. Se consideran a la vez como información demográfica: edad, sexo, estado civil, así como, red social que se utiliza para el envío de mensajes, fotos y videos.

\section{Plan de prueba}

Para llevar a cabo la validez de este instrumento se consideró una validez de contenido, es decir, se revisó la literatura para conocer a fondo las actitudes relacionadas hacia cada una de las dimensiones evaluadas: conductas sexuales de riesgo y motivos. Para cumplir con una validez más estricta se aplicó el instrumento a 263 participantes.

\section{Tratamiento estadístico}

Una vez capturados los datos en el programa estadístico IBM SPSS Statistics versión 20 para Windows en inglés, se dio el tratamiento estadístico pertinente:

1. Análisis de frecuencias, medidas de tendencia central y dispersión para cada reactivo.

2. Análisis de discriminación y direccionalidad para todos los reactivos.

3. Análisis factorial para cada dimensión.

4. Análisis de confiabilidad interna Alfa de Cronbach por cada factor para todas las dimensiones.

Con el objetivo de conocer las distribuciones de tendencia central y dispersión se obtuvieron la media, desviación estándar, sesgo y la curtosis de todos los reactivos.

\section{RESULTADOS}

Dado que todos los reactivos cumplieron con las pruebas de distribución, se decidió incluirlos en la prueba 
factorial. Se realizó un análisis factorial de componentes principales con rotación oblicua. Se encontraron dos factores con peso propio mayor a 1 (Tabla 1). La dimensión de "conductas sexuales de riesgo" quedó compuesta por 14 reactivos que explican el $75.086 \%$ de la varianza con una confiabilidad de .962 , dividido en dos factores, el primero explica el $67.150 \%$ de la varianza y con una confiabilidad de .950 , el segundo factor explica el $7.936 \%$ de la varianza con una confiabilidad de .924 .

Tabla 1. Factores de la dimensión "conductas sexuales de riesgo". Peso del factor, porcentaje de varianza explicado y alfa del factor y dimensión.

\begin{tabular}{|c|c|c|c|c|c|c|}
\hline \multicolumn{2}{|r|}{ FACTORES } & 1 & 2 & Peso & \%V.E. & Alfa \\
\hline \multirow{7}{*}{ 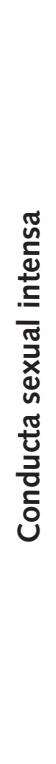 } & $\begin{array}{l}\text { 9. Envié fotografías personales manteniendo relaciones } \\
\text { sexuales con alguien a mi pareja formal. }\end{array}$ & .968 & & \multirow{7}{*}{9.401} & \multirow{7}{*}{67.150} & \multirow{7}{*}{.950} \\
\hline & $\begin{array}{l}\text { 10. Envié fotografías personales masturbándome a mi } \\
\text { pareja formal. }\end{array}$ & .961 & & & & \\
\hline & $\begin{array}{l}\text { 13. He recibido videos con contenido sexual de mi } \\
\text { pareja formal. }\end{array}$ & .910 & & & & \\
\hline & $\begin{array}{l}\text { 12. He enviado videos personales con contenido sexual } \\
\text { a mi pareja formal. }\end{array}$ & .872 & & & & \\
\hline & $\begin{array}{l}\text { 2. Utilicé nombres sexualizados o sugerentes en chats } \\
\text { en línea con mi pareja formal. }\end{array}$ & .788 & & & & \\
\hline & $\begin{array}{l}\text { 14. He mantenido cibersexo en vivo a través de } \\
\text { videoconferencias (por ejemplo, vía Skype) con mi } \\
\text { pareja formal. }\end{array}$ & .617 & & & & \\
\hline & $\begin{array}{l}\text { 11. He enviado fotografías personales con poses } \\
\text { sugerentes pero con ropa a mi pareja formal. }\end{array}$ & .488 & & & & \\
\hline \multirow{9}{*}{ 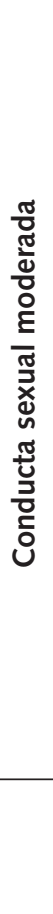 } & $\begin{array}{l}\text { 4. Mantuve pláticas sexuales en línea con mi pareja } \\
\text { formal. }\end{array}$ & & .980 & \multirow{7}{*}{1.111} & \multirow{7}{*}{7.936} & \multirow{7}{*}{.92} \\
\hline & $\begin{array}{l}\text { 6. Recibí fotografías con contenido sexual de mi pareja } \\
\text { formal. }\end{array}$ & & .666 & & & \\
\hline & $\begin{array}{l}\text { 8. Envié fotografías personales con poca ropa a mi } \\
\text { pareja formal. }\end{array}$ & & .634 & & & \\
\hline & $\begin{array}{l}\text { 5. Envié fotografías personales con contenido sexual a } \\
\text { mi pareja formal. }\end{array}$ & & .623 & & & \\
\hline & $\begin{array}{l}\text { 1. Recibí solicitudes para tener alguna conducta sexual } \\
\text { por internet con mi pareja formal. }\end{array}$ & & .617 & & & \\
\hline & 3. Mantuve cibersexo con mi pareja formal. & & .533 & & & \\
\hline & $\begin{array}{l}\text { 7. Envié fotografías personales con desnudos a mi } \\
\text { pareja formal. }\end{array}$ & & .522 & & & \\
\hline & Media & 2.787 & 2.192 & & \multirow{2}{*}{75.086} & \multirow{2}{*}{.962} \\
\hline & Desviación estándar & 1.171 & 1.214 & & & \\
\hline
\end{tabular}


Al realizar una prueba de correlación entre los factores obtenidos podemos observar una correlación positiva entre el factor conductas sexuales intensas (CSI) y el factor conductas sexuales moderadas (CSM). Esto significa que a mayores conductas sexuales intensas, mayores conductas sexuales moderadas por parte de los participantes (Tabla 2).

Tabla 2. Correlaciones entre el factor conductas sexuales intensas formal y conductas sexuales moderadas formal

\begin{tabular}{c|c|c}
\multicolumn{2}{|l|}{ CSIF } & \multicolumn{1}{l}{ CSLF } \\
\hline CSIF & 1.000 & $.834^{* *}$ \\
\hline CSLF & & 1.000
\end{tabular}

\section{Motivos}

Como complemento a la dimensión de "conductas sexuales de riesgo" se creó la dimensión "motivación hacia el sexo en línea”. Se realizó un análisis factorial de componentes principales con rotación oblicua (Tabla 3). Se encontró un factor con peso propio mayor 1. La dimensión de "motivación hacia el sexo en línea" quedó compuesta por seis reactivos que forman un sólo factor que explica el $68.930 \%$ de la varianza con una confiabilidad de .90 .

Tabla 3. Factores de la dimensión "motivación hacia el sexo en línea". Peso del factor, porcentaje de varianza explicado y alfa del factor y dimensión.

\begin{tabular}{|c|c|c|c|c|c|}
\hline & FACTORES & 1 & Peso & \%V.E. & Alfa \\
\hline \multirow{8}{*}{ 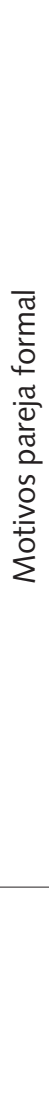 } & $\begin{array}{l}\text { 18. He enviado algún mensaje de texto, o imagen, o video } \\
\text { con contenido sexual para iniciar el acto sexual con mi } \\
\text { pareja formal. }\end{array}$ & .868 & \multirow{6}{*}{4.136} & \multirow{6}{*}{68.930} & \multirow{6}{*}{.909} \\
\hline & $\begin{array}{l}\text { 19. He enviado algún mensaje de texto, o imagen, o video } \\
\text { con contenido sexual por aburrimiento a mi pareja formal. }\end{array}$ & .851 & & & \\
\hline & $\begin{array}{l}\text { 16. He enviado algún mensaje de texto, o imagen, o video } \\
\text { con contenido sexual porque me lo solicitó mi pareja } \\
\text { formal. }\end{array}$ & .843 & & & \\
\hline & $\begin{array}{l}\text { 17. He enviado algún mensaje de texto, o imagen, o video } \\
\text { con contenido sexual porque prefiero que el contacto sea } \\
\text { virtual que real con mi pareja formal. }\end{array}$ & .838 & & & \\
\hline & $\begin{array}{l}\text { 20. He enviado algún mensaje de texto, o imagen, o video } \\
\text { con contenido sexual porque mi relación era a distancia con } \\
\text { mi pareja formal. }\end{array}$ & .807 & & & \\
\hline & $\begin{array}{l}\text { 15. He enviado algún mensaje de texto, o imagen, o video } \\
\text { con contenido sexual para coquetear con mi pareja formal. }\end{array}$ & .770 & & & \\
\hline & Media & 2.458 & & \multirow{2}{*}{68.930} & \multirow{2}{*}{.909} \\
\hline & Desviación estándar & 1.170 & & & \\
\hline
\end{tabular}




\section{DISCUSIÓN Y CONCLUSIONES}

Para la construcción de los instrumentos de conductas sexuales de riesgo en usuarios de redes sociales virtuales se llevó a cabo una revisión literaria de instrumentos previos, no se contó con ningún instrumento en español por lo cual nos basamos, en un principio, en el trabajo de Delmonico y Miller (2003) ya que al realizar su "The Internet Sex Screening Test" obtuvieron un instrumento con cinco factores, siendo Online Sexual Behavior uno de los factores de nuestro interés por la presencia de reactivos asociados con las respuestas obtenidas en los grupos focales tal como "utilizo nombres sexualizados o sugerente en chats en línea" lo cual está fuertemente relacionado con la idea de ser menos tímido y formar una imagen tal vez falsa a través del internet.

Drouin y Landgraff (2012) trabajaron con la dimensión de prevalencia del sexting. Tomando como referencia esta dimensión y sus reactivos, en nuestro caso trabajamos con reactivos como "mantuve pláticas sexuales en línea", "envié fotografías personales con contenido sexual" y "mantuve cibersexo en vivo a través de videoconferencias (por ejemplo vía Skype)”. Así mismo, trabajaron con una dimensión llamada contenido de los mensajes de fotos y videos, a través de una tabla de frecuencias, en nuestro caso, redactamos los reactivos "envié fotografías personales con desnudos; manteniendo relaciones sexuales con alguien; masturbándome y con poses sugerentes pero con ropa”. Esto nos dirigió al motivo por el cual las personas llevaban a cabo este tipo de conductas con reactivos como "envié algún mensaje de texto, o imagen, o video con contenido sexual para coquetear"; "porque mi pareja me lo so-

\section{REFERENCIAS BIBLIOGRÁFICAS}

Asociación Mexicana de Internet. AMIPCI; 2014 http:// www.amipci.org.mx/.

Baumgartner, S.E; Valkenburg, P.M. \& Peter, J. (2010). "Unwanted online sexual solicitation and risky sexual online behavior across the lifespan." Journal of Applied Developmental Psychology. 31, 439-447 en http://dx.doi.org/10.1016/j.appdev.2010.07.005

Benotsch, E.G; Kalichman, S. \& Cage. (2002). "Men Who Have Met Sex Partners via the Internet: Prevalence, licitó"; "porque prefiero que el contacto sea virtual más que real"; "para iniciar el acto sexual"; por aburrimiento y; porque "mi relación era a distancia". Mientras que Baumgartner, Valkenburg y Peter (2010) estudiaron la dimensión sobre la comunicación por internet, ya que consideran que entre mayor comunicación por la web, mayores son las insinuaciones sexuales no deseadas y las conductas sexuales con riesgo. En nuestro caso, se trabajó con una tabla de frecuencias para saber el uso que hacen de las diferentes redes sociales para el envío de mensajes con contenido sexual.

De esta manera, el instrumento mostró dos dimensiones, la primera formada por conductas sexuales intensas y la segunda de moderadas, siendo la media más alta para el factor de conductas moderadas. Es en este factor donde los participantes hacen referencia a enviar fotografías con contenido sexual o fotografías sugerentes pero con ropa. Mientras que la segunda dimensión hace referencia a recibir fotografías, mantener pláticas y cibersexo en línea. La dimensión de motivos está constituida por un solo factor que hace referencia a enviar fotografías o mensajes de desnudos porque la pareja lo ha solicitado, por aburrimiento o por distanciamiento. Estas conductas hacen referencia a aspectos relacionados a la búsqueda de conductas sexuales en línea con la pareja formal. Los participantes en el estudio afirmaron haber tenido este tipo de conductas en al menos una ocasión con su pareja formal.

Finalmente, las dos escalas muestran índices de confiabilidad y validez adecuados. Se recomienda ampliar la muestra y probarlo en otras poblaciones para aumentar su validez.
Predictors, and Implications for HIV Prevention." Archives of Sexual Behavior, 31(2), 177-183.

Bogaert, A. F. (2001). "Personality, individual differences, and preferences for the sexual media." Archives of the sexual behavior, 30, 29-53.

Crimmins, D.M. \& Siegfried-Spellar, K.C. (2014). "Peer attachment, sexual experiences, and risky online behaviors as predictors of sexting behaviors among undergraduate students." Computers in Human 
Behavior, 32, 268-275 en http://dx.doi.org/10.1016/j. chb.2013.12.012

Delmonico, D.L. \& Miller, J.A. (2003). "The Internet Sex Screening Test." Sexual and Relationship Therapy, 18, 261-276.

Drouin, M. \& Landgraff, C. (2012). “Texting, Sexting, and Attachment in College Students' Romantic Relationships." Computers in Human Behavior, 28(2), 444-449. http://dx.doi.org/10.1016/j.chb.2011.10.015

Drouin, M., Vogel, K.N., Surbey, A., \& Stills, J.R. (2013). "Let's talk about sexting, baby: Computer-mediated sexual behaviors among young adults. Computers in Human Behavior." Article in press en http://dx.doi. org/10.1016/j.chb.2012.12.030

Fisher, W.A. \& Barak, A. (1991). "Pornography, erotic, and behavior: More questions than answers." International Journal of Law and Psychiatry, 14, 6583. http://dx.doi.org/10.1016/0160-2527(91)90025-I

Gordon, Serena. "Teens Divulge Risky Behavior on Social Networking Sites-US News and World Report." Health News Articles-US News Health. 6 Jan. 2009.

Kujath C.L. (2011). "Facebook and MySpace: Complement or Substitute for Face-to-Face Interaction?" CyberPsychology, Behavior \& Social Networking, 4(1/2), 75-78. DOI: 10.1089/cyber.2009.0311

Lau, W \& Yuen, A. (2013). 'Adolescents' risky online behaviours: The influence of gender, religion, and parenting style." Computers in Human Behavior, 29(6), 2690-2696. http://dx.doi.org/10.1016/j.chb. 2013.07.005
Malamuth, N.M., Addison, T. \& Koss, M. (2001). Pornography and sexual aggression. Orlando, FL: Academic Press.

Mitchell, K.J; Finkelhor, D. \& Wolak, J. (2001). “Risk factors for and impact of online sexual solicitation of youth." JAMA, the Journal of the American Medical Association, 285, 3011-3014.

Mitchell, K.J; Finkelhor, D. \& Wolak, J. (2007). "Youth internet users at risk for the most serious online sexual solicitations." American Journal of Preventive Medicine, 32, 532-537. DOI: 10.1016/j.amepre.2007. 02.001

Mitchell, K.J; Finkelhor, D. \& Wolak, J. (2007a). “Online requests for sexual pictures from youth: Risk factors and incident characteristics." The Journal of Adolescent Health, 41, 196-203. http://dx.doi.org/ 10.1016/j.jadohealth.2007.03.013

Wright, P.J. \& Randall, A.K. (2012). "Internet pornography exposure and risky sexual behavior among adult males in the United States." Computers in Human Behavior, 28, 1410-1416. http://dx.doi.org/10.1016/j. chb.2012.03.003

Ybarra, M.L; Espelage, D.L. \& Mitchell, K.J. (2007). “The co-occurrence of internet harassment and unwanted sexual solicitation victimization and perpetration: Associations with psychosocial indicators. Journal of Adolescent Health, 41, S31-41. 